\title{
Assessment of a test of renal viability
}

\author{
G WILLIAMS，T N D PEET, R J HAMSHERE
}

British Medical fournal, 1976, 2, 75-77

\section{Summary}

A test of renal viability using the uptake of ${ }^{125}$ iodohippurate by kidney biopsy specimens has been developed. It is applicable to all kidneys irrespective of the method of storage. The uptake of ${ }^{125}$ I-iodohippurate in experimental kidneys correlated well with warm or cold ischaemia time and subsequent renal function. The test was used for human cadaver kidneys offered for transplantation and there was good correlation between iodohippurate uptake and warm ischaemic time. With this test, prediction of renal function was accurate in $8^{\circ}{ }^{\circ}$ of human cadaver kidneys transplanted. Pulsatile perfusion had no effect on cadaver kidneys as measured by this test.

\section{Introduction}

Almost a third of the cadaver kidneys transplanted in the United Kingdom never function or function poorly. ${ }^{1}$ Excluding technical cr.uses, the usual cause of failure after transplantation is premortem hypotension, which is often inadequately recorded or 1.ot known about at all. ${ }^{2}$ It is irrational and possibly unethical to subject a recipient to organ transplantation unless there is a good chance that the organ will function. When live donor kidneys are used there is a striking contrast in subsequent functon compared with the usual cadaver kidney transplants, and early failure is rare.

A reliable test of renal viability would therefore decrease the transplant failure rate. Such a test should be simple; able to be completed within the time taken for the preoperative donorrecipient direct cytotoxic cross match and preparation of the operating theatre; and applicable regardless of the kidney preservation method.

\section{Methods}

In developing such a test a series of experiments were performed on rat kidney slices that determined the optimum slice weight and thickness, the oxygen requirements, and period of inclibation with iodohippurate. ${ }^{34}$

${ }^{125}$ I-iodohippurate $5 \mu \mathrm{Ci}$ is added to a buffered medium made up of $43.5 \mathrm{ml}$ of $0.3-\mathrm{M}$ sodium chloride, $10 \mathrm{ml}$ of $0.1-\mathrm{M}$ sodium phosphate

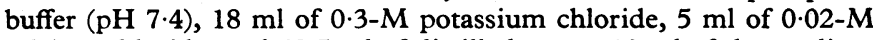
calcium chloride, and $48.5 \mathrm{ml}$ of distilled water. $10 \mathrm{ml}$ of the medium is transferred to a $25-\mathrm{ml}$ conical flask maintained at $25^{\circ} \mathrm{C}$ in a water bath and oxygenated with $100 \%$ oxygen $(3-500 \mathrm{ml} / \mathrm{min})$ before and while the kidney slices are immersed in the medium. The slices (0.3-0.4 $\mathrm{mm}$ thick) are cut with a microtome from kidney biopsy specimens (30-40 mg) obtained with a biopsy tool (fig 1) and the slices are immersed without delay for one hour. They are then removed from the medium and, after blotting, are transferred to small plastic

\footnotetext{
Urology and Transplantation Unit, Department of Surgery, Royal Postgraduate Medical School, Hammersmith Hospital, London W12 0HS

G WILLIAMS, MB, FRCS, senior registrar

T N D PEET, MB, FRCS, registrar

R J HAMSHERE, MB, FRCS, research registrar
}

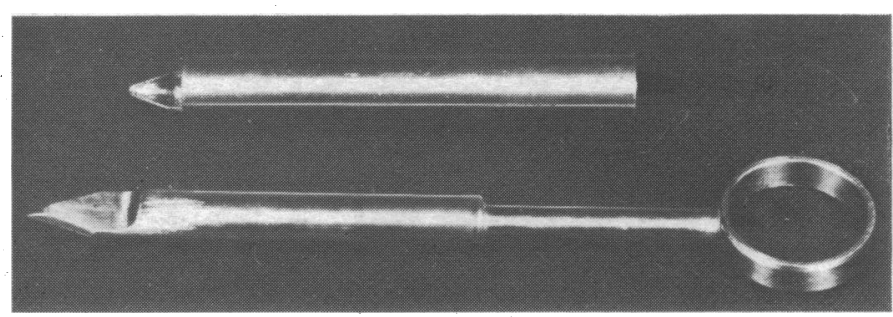

FIG 1-Renal biopsy tool consisting of outer sheath with terminal cutting edge and inner knife blade.

phials (Gammavial, Koch Light Laboratories Limited) for determination of radioactivity (counts/min). It is important to push the slices down on to the bottom of the phials so that there is a degret of constancy between the geometry of the slices and the scintillation counter used to detect the radioactivity. The radioactivity of the medium used for incubating the slices is separately determined by pipetting three drops into pre-weighed phials. Subtracting the weights of the respective phials from those that contained the slices and those containing the medium, the radioactivity per gram of biopsy specimen and per gram of medium is calculated. The ratio between the two, slice:medium, is designated the $S: M$ ratio.

Three animal studies were performed, using female Wistar rats weighing $250 \mathrm{~g}$. Three studies using human kidneys were also performed.

\section{Experiments and results}

EXPERIMENT 1: VARIABLE PERIODS OF WARM ISCKAEMIA

Three groups of eight rats were used. Under ether anaesthesia the left kidney was exposed and fully mobilised from surrounding tissues. The renal pedicle was occluded by a rubber band for 30 minutes in one group, 60 minutes in another, and 90 minutes in the third group. The rubber band was removed and the rats allowed to recover. Three weeks later contralateral nephrectomy was performed. The blood urea was measured twice weekly for three weeks after the nephrectomy or until the animal died.

The group subjected to a: 30-minute warm ischaemia time showed no significant rise in blood urea levels and only one animal died (fig 2). When the warm ischaemia time was prolonged to 60 minutes and

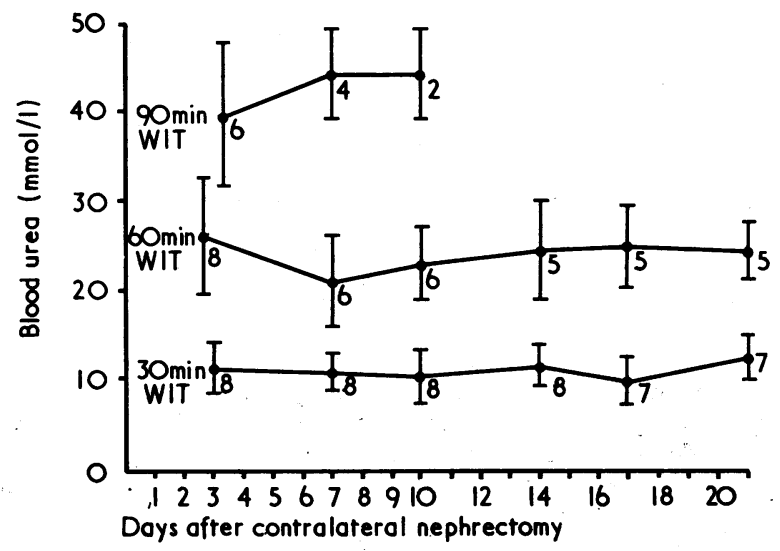

FIG 2-Rat blood urea levels ( \pm 1 .SD) after various periods of warm ischaemia time (WIT) and contralateral nephrectomy. Numbers of rats surviving at each stage are shown.

Conversion: SI to traditional units-Urea: $1 \mathrm{mmol} / 1 \approx 6 \mathrm{mg} / 100 \mathrm{ml}$. 
above there was a prolonged persistent rise in blood urea levels associated with reduced survival of the animals.

\section{EXPERIMENT 2: S:M RATIO AND WARM ISCHAEMIA TIME}

Both kidneys were removed from six groups of four rats and stored at $37^{\circ} \mathrm{C}$ for varying periods of warm ischaemia. The $S: M$ ratio of all kidneys was then calculated. The results in fig 3 show that prolongation of warm ischaemia was associated with a decline in the $\mathrm{S}: \mathrm{M}$ ratio.

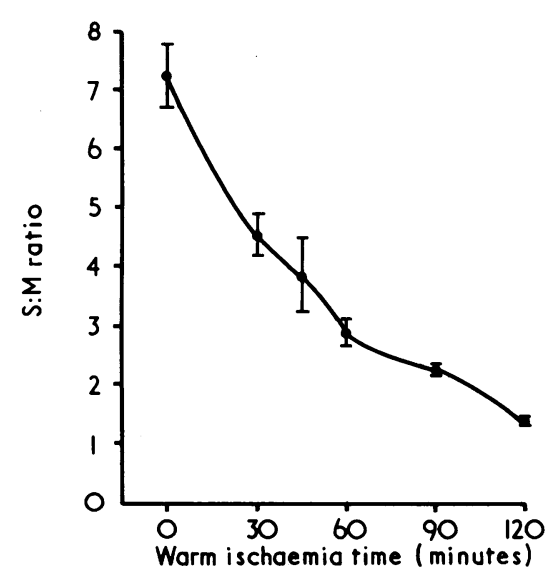

FIG 3-S:M ratio of rat kidney biopsy specimens submitted to known periods of warm ischaemia.

\section{EXPERIMENT 3: S:M RATIO AND COLD ISCHAEMIA TIME}

Both kidneys were removed from five groups of rats and stored for 30 minutes in buffered saline at $37^{\circ} \mathrm{C}$ and then removed to cold buffered saline at $4^{\circ} \mathrm{C}$ for periods up to 12 hours and the $\mathrm{S}: \mathrm{M}$ ratio calculated. Prolongation of the cold ischaemia time beyond four hours was associated with a reduction in the $S: M$ ratio (fig 4), but up to 12 hours' storage did not cause the $S: M$ ratio to fall below 3 .

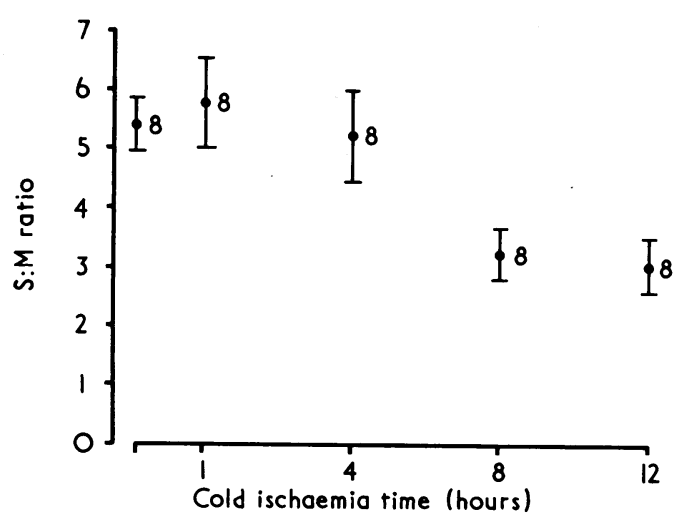

FIG 4-S:M ratio of rat kidney biopsy specimens with 30 minutes' warm ischaemia and varying cold ischaemia times. Numbers in each group are shown.

\section{HUMAN STUDIES}

Three separate groups of human kidneys were studied.

Single biopsy specimens were obtained from five kidneys from patients undergoing surgery for calculous disease. This group with no warm ischaemia were considered controls. The $S: M$ ratios of these ranged from 5 to 6.9 (fig 5 ).

Single biopsy specimens were also obtained from 27 cadaver kidneys offered for transplantation, and the $S: M$ ratio was calculated and the results correlated with subsequent function. The $S: M$ ratios of this group ranged from 1.0 to 8.0 . Three of these kidneys were not transplanted because of vascular abnormalities. Kidneys with an $S: M$

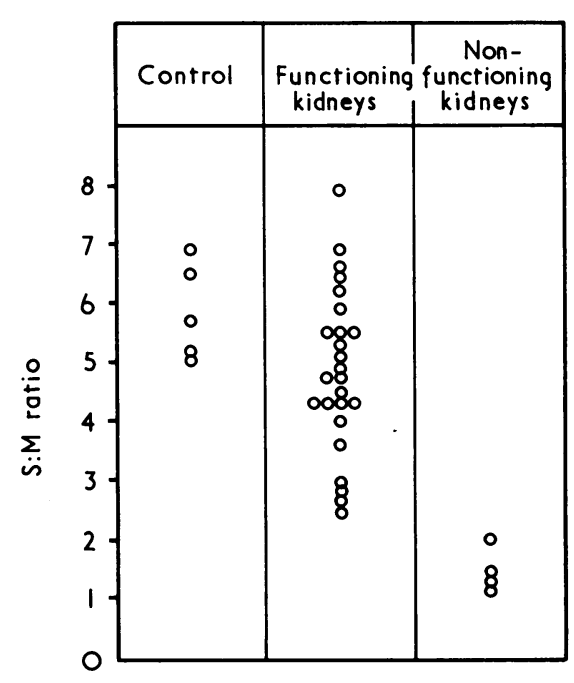

FIG 5-S:M ratios of human kidney biopsy specimens showing eventual outcome after transplantation.

ratio below 2 did not function whereas all kidneys with an $S: M$ ratio ov greater than 3 functioned (fig 5). The four kidneys with $S: M$ ratios 음 between 2 and 3 functioned-one 24 hours after transplantation and the other three after prolonged periods of tubular necrosis (18 to $26 \vec{\circ}$ days). All kidneys with $S: M$ ratios greater than 3 functioned within 12 days.

Five kidneys were perfused on an AB-Gambro perfusion machine. Initial $S: M$ ratios ranged from $4 \cdot 3$ to $6 \cdot 4$, and on further biopsy these figures had not changed during periods of perfusion from six to 14 hours (fig 5). All these kidneys functioned within five days of transplantation.

\section{Discussion}

Several tests of kidney viability have been described. The value of most of these is diminished by the need for continuous pulsatile perfusion, ${ }^{5}$ which is not commonly available in this country. Predicting viability by estimating adenine nucleotide content has been examined ${ }^{6}$ but has not been applied to man. Estimating the potassium:sodium ratio in kidney biopsy specimens has been advocated by Sells et al, ${ }^{3}$ but animal $\dot{0}$ studies $^{8}$ could not correlate the potassium:sodium ratio with warm ischaemia time or ability of the kidney to support life.

Experiment 1 showed that prolonged warm ischaemia causes severe renal damage, as shown by the rising blood urea levels and $ㅇ$ early death of animals subjected to a warm ischaemia time of over $N$ 60 minutes. The second experiment showed excellent correlation of between increasing warm ischaemia time and declining $S: M$ ratio. The third animal experiment examined the effect of increasing cold ischaemia. This showed a fall in the $S: M$ ratio 0 to three at 12 hours, and we predict that these kidneys would still $\underset{\omega}{N}$ have been viable. Hall et $a^{9}$ have shown that prolonging the cold ischaemia time beyond 12 hours is associated with a higher 0 incidence of primary non-function.

From these data we predicted that a kidney with an $S: M \stackrel{\mathscr{P}}{+}$ ratio of over 3 will be viable and should function after transplantation. A kidney with an $S: M$ ratio below 3 will have severe renal damage and will function poorly or not at all.

When the test was applied to human kidneys the control series $\stackrel{\mathbb{Q}}{\Omega}$ with no warm ischaemia time had an $S: M$ ratio between 5 and $q$ 6.9 , which compared well with that of the similar animal group. The slightly lower range in the human group may have been because these specimens came from diseased kidneys.

The observation of only moderate correlation between warm ischaemia time and the $S: M$ ratio $(r=-0.43)$ in cadaver kidneys (fig 6) probably reflects the inaccuracies of recording antemortem hypotension. The $S: M$ ratio seems to be of greater predictive value in determining renal viability than the recorded warm 


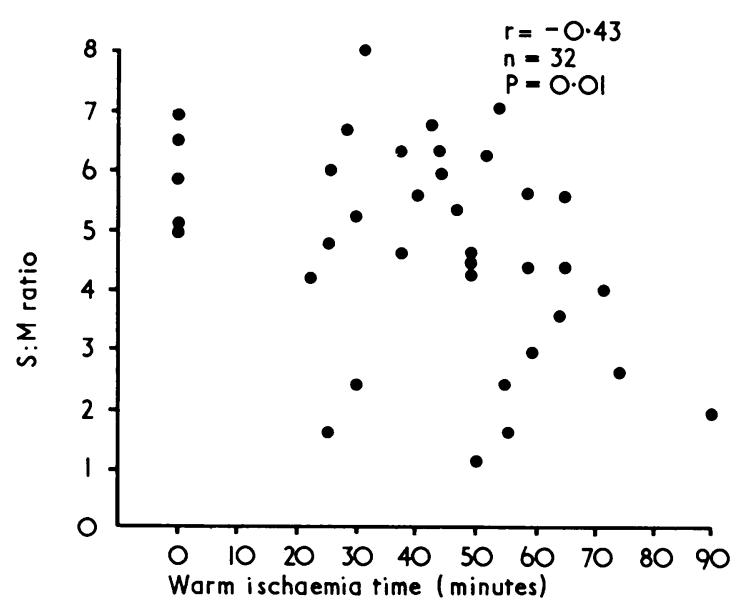

FIG 6-S:M ratio of all human kidney biopsy specimens showing correlation with warm ischaemia time.

ischaemia time. All kidneys with an $S: M$ ratio over 3 have functioned. Four cadaver kidneys with an $S: M$ ratio under 2 did not function, and on the basis of animal studies this was predicted before transplantation. As the whole technique is in an experimental stage we could not deny the use of these kidneys for transplantation. The critical range of $S: M$ ratio where function is less easy to determine is between 2 and 3. Four kidneys in this range worked, one 24 hours after transplan- tation and the other three after prolonged periods of tubular necrosis. Biopsy of two of these 14 days after transplantation showed extensive tubular atrophy and patchy cortical necrosis. Further use of the test is required to evaluate the critical area between 2 and 3 .

${ }^{125}$ I-iodohippurate uptake by donor kidney slices can be applied to kidneys stored on ice or by pulsatile perfusion, or while still in situ. Preservation by pulsatile perfusion of human kidneys did not seem to cause either improvement or deterioration in the $S: M$ ratio. This contrasted with the observation that prolonged cold storage of rat kidneys caused a deterioration in the $S: M$ ratio.

We acknowledge the help and advice given by Professor R Shackman. Requests for reprints should be addressed to Mr G Williams Department of Surgery, Royal Postgraduate Medical School, Hammersmith Hospital, Du Cane Road, London W12 0HS.

\section{References}

${ }^{1}$ National Organ Matching Report. Bristol, October, 1973.

2 Carroll, R N P, Chisholm, G D, and Shackman, R, Lancet, 1969, 2, 551.

${ }^{3}$ Hamshere, R J, and Lipscombe, J, unpublished data.

4 Slapak, M, and Rodgers, S, Transplantation Proceedings, 1975, 3, Suppl No 1, p 569.

${ }^{5}$ Baxby, K, et al, Lancet, 1974, 2, 977.

${ }^{6}$ Calman, K C, and Bell, P R F, British fournal of Surgery, 1973, 60, 322.

' Sells, R A, McLoughlin, R A, and Tyrrell, I, British fournal of Surgery, $1974,61,326$

${ }^{8}$ Rogers, S, and Slapak, M, British fournal of Surgery, 1974, 61, 98.

9 Hall, C L, et al, British Medical fournal, 1975, 3, 667.

\title{
Superficial carcinoma of the stomach
}

\author{
G MACHADO, J D DAVIES, A J C TUDWAY, P R SALMON, A E READ
}

British Medical fournal, 1976, 2, 77-79

\section{Summary}

Nine cases of superficial gastric carcinoma have been detected with upper gastrointestinal endoscopy in Bristol in the past two years. This contrasted with only six cases found from postoperative gastrectomy specimens examined in the previous eight years. It is often difficult to distinguish a superficial carcinoma from a benign ulcer, and endoscopic diagnosis is effective only if multiple biopsy specimens are taken. Endoscopy should also be repeated and multiple specimens taken until the lesion has healed; even malignant ulcers may heal, and any healed area that is depressed with interrupted mucosal folds should be suspected of malignancy. The endoscopic and histological appearances, the age of the patients, and the clinical behaviour of the disease resembled descriptions of the disease, principally from Japan. Superficial gastric carcinoma is probably underdiagnosed in Britain.

\footnotetext{
University Departments of Medicine and Histopathology, Bristol Royal Infirmary, Bristol BS2 8HW

G MACHADO, MD, consultant assistant professor, Universidade Federal do Rio de Janeiro

J D DAVIES, MD, MRCPATH, consultant senior lecturer in pathology

A J C TUDWAY, MB, BCHIR, lecturer in pathology

P R SALMON, MRCP, consultant senior lecturer in medicine

A E READ, MD, FRCP, professor of medicine
}

\section{Introduction}

Japanese experience has shown a remarkable five-year survival rate of over $90^{\circ}{ }_{0}$ in surgically treated patients with carcinoma of the stomach limited to the mucosa or submucosa (superficial carcinoma). ${ }^{2}$ This is in striking contrast to an overall survival rate of about $15^{\circ}$ in the United Kingdom ${ }^{3}{ }^{4}$ and the United States. ${ }^{5}$ This low survival rate is probably due to the infrequent detection of superficial gastric carcinoma. ${ }^{6-8}$ Over a third of cases in Japan are now detected at this stage. ${ }^{12}$ The Japanese relate the increased diagnosis of superficial gastric carcinoma to the widespread use of endoscopic examination with biopsy. In view of its potential importance we report our experience of the changing incidence of superficial carcinoma of the stomach in a British centre.

\section{Methods}

Two groups of patients were studied. The material received in the university department of histopathology from all patients submitted to gastric resection between January 1965 and May 1975 was reviewed. The second group included all patients referred to the digestive endoscopy unit, university department of medicine, Bristol Royal Infirmary, from its establishment in December 1969 to May 1975.

Endoscopic examinations were performed as reported ${ }^{9}$ with the ACMI F-8 fibrescope, and biopsy specimens were taken when the appearances suggested malignancy or when ulceration or polyps were found. The paraffin-embedded biopsy tissue obtained at gastroscopy was treated as described, ${ }^{10}$ and the stomachs resected between 1973 and 1975 were examined according to a modification of the method of Mochizuki."11 The macroscopic classification of superficial gastric carcinoma laid down in 1962 by the Japanese Society for Gastroenterological Endoscopy ${ }^{12}$ was adopted (see figure). 